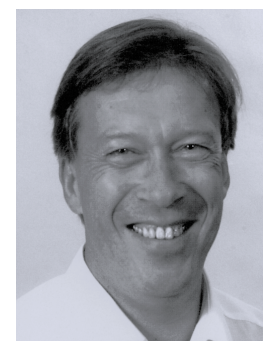

Christian Deckert

\title{
Psychiatrische Patienten zwischen Hausarzt und Facharzt
}

\author{
Christian Deckert, Hamburg
}

psychoneuro 2003; 29 (11): 509-512

$\mathrm{P}$ sychische Erkrankungen gewinnen in der medizinischen Versorgung der Bevölkerung zunehmend an Bedeutung (1). Einer komplexen Diagnostik auf neuropsychiatrischem Fachgebiet müssen dynamisch flexible Therapieantworten folgen, was durch die bisherige unzureichende Übermittlung von Therapieleitlinien an den Hausarzt als Ersttherapeut problematisch erscheint. Nur gemeinsame Kenntnisse hierüber und ausreichende Information zwischen Hausarzt und Nervenarzt bei Behandlungsbeginn besitzen eine hohe Effektivität hinsichtlich Remission und Prognose der Erkrankung und Lebensqualität des Patienten.

Fehlt ein gemeinsames

Behandlungskonzept?

Bisher stehen verlässliche Datenquellen, die belastbare statistische Informationen über das Überweisungsverhalten bieten, noch aus. Bei einer Durchsicht in eigener Praxis nach Anfragen der Hausärzte zu neuro-psychiatrischen Krankheitsbildern stellt sich die Rangliste nach der Häufigkeit wie in den Tabellen 1 und 2 gezeigt dar.

Die Anfragen äußern sich in Form von Telefonaten, gemeinsamen Konsilen und der Bitte um Hausbesuche beim Kranken. Hier

Verlässliche Datenquellen, die belastbare statistische Informationen zum Überweisungsverhalten zwischen Hausärzten und Fachärzten bieten, stehen zurzeit noch nicht zur Verfügung. In dieser Arbeit werden die subjektiven Erfahrungen eines Nervenarztes zu Anfragen der Hausärzte zu neuro-psychiatrischen Krankheitsbildern vorgestellt. Dabei muss festgestellt werden, dass der Nervenarzt häufig erst spät zur Diagnostik und Therapie hinzugezogen wird, obwohl beide Arztgruppen gut von einer besseren Zusammenarbeit profitieren könnten. Vernetzte und interdisziplinäre Fortbildung sind dringend erforderlich, damit das Wissen aus Universität, fachärztlicher und allgemeinmedizinischer Kompetenz zusammenfließen kann.

hat sich über viele Jahre ein Management entwickelt, dass an die Logistik der alltäglichen ambulanten nervenärztlichen Arbeit besondere Anforderungen stellt. Ihre Bewältigung setzt u.a. einen vertrauten, kollegialen Kontakt zu den Hausärzten voraus. Nachfolgend werden ihre Anfragen kommentiert.

Psychiatrische Fragestellungen nach Diagnosen

- Schizophrenie, Psychose: Schizophrene Patienten werden aktuell von Hausärzten in der Regel zur stationären Therapie eingewiesen, bei weiterer ambulanter Behandlung obliegt das Therapieregime dem Nervenarzt $(2,3)$. Ähnliches gilt bei der Abklärung von psychotischen Krankheitsbildern. Ihre Früherkennung stellt die Hausärzte vor große Probleme

- Schlafstörung: Häufig werden hier die betroffenen Patienten symptomatisch anbehandelt und bei Non-Response zur weiteren Abklärung vorgestellt

- Zyklothymie bzw. bipolare Störung: Hier gilt Ähnliches wie bei der Abklärung von psychotischen Krankheitsbildern

- Essstörung: Häufig erfolgt die Zuweisung in stationäre Spezialabteilungen, nur selten eine ambulante Vorabklärung durch den Nervenarzt

- Borderline-Persönlichkeitsstörung: Der Komplexität des Krankheitsbildes entsprechend bestehen große Unsicherheiten in der Diagnostik mit häufigen stationären Einweisungen

- Phobie: Auch langwierige Verläufe bleiben diagnostisch oft unerkannt, die Vorstellung erfolgt aber primär mit der Bitte um Erstellung von Therapiekonzepten, weniger zur Diagnosestellung

- Psychosomatose/Somatisierungs- 
störung: Meist wird eine langwierige und aufwändige Diagnostik mit Beteiligung mehrerer somatischer Fachbereiche, vorrangig Orthopädie, Labor und Bildgebung durchgeführt, eine nervenärztliche Beurteilung erfolgt meist zuletzt

- Schmerz: Durch ausschließlich symptomatischen Einsatz von Analgetika kommt es schnell zu einem Missbrauch

- Angststörungen: Ein mehrjähriger unspezifisch behandelter Symptomverlauf führt durch sein frustranes Therapieergebnis leider häufig nur zufällig zum
Nervenarzt. Trotz mitunter schwieriger Differenzialdiagnostik lässt sich dort die Erkrankung in der Regel zuverlässig erkennen und behandeln (4)

- Depression: Das weit reichende Feld der Differenzialsymptomatik führt häufig zu diagnostischen Unsicherheiten und sehr komplexen Therapieeinschätzungen. Hier wird eine der wichtigsten Aufgaben deutlich, die Diagnostik der häufigsten psychiatrischen Erkrankung und deren Verlauf gemeinsam zwischen Hausarzt und Nervenarzt zu koordinieren

- Sucht: Unsicherheiten in Thera-

\section{Tab. 1 Häufigste Anfragen der Hausärzte zu psychiatrischen Krankheitsbildern}

nach Diagnosen
- Schizophrenie
- Psychose
- Schlafstörung
- Zyklothymie bzw. bipolare
Störung
- Essstörung
- Borderline-Persönlichkeitsstörung
- Phobie
- Psychosomatose
- Schmerz
- Angst
- Depression
- Sucht
- Aggression
- Demenz
- Zwang

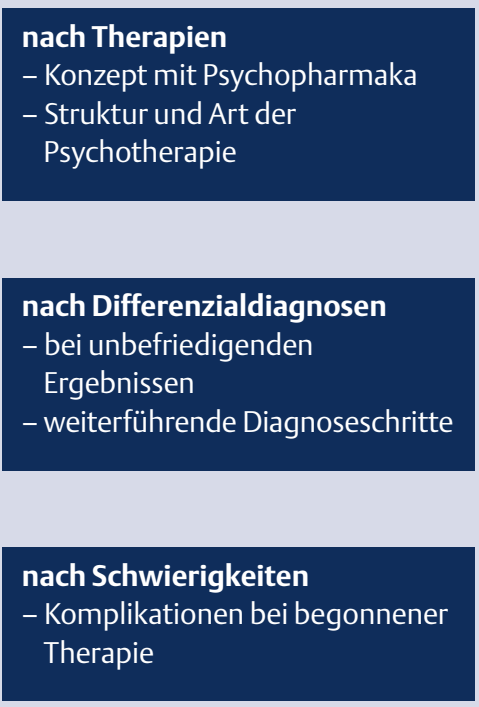

Tab. 2 Häufigste Anfragen der Hausärzte zu neurologischen Krankheitsbildern

nach Diagnosen

- Schwindel

- Wirbelsäulensyndrom

- Encephalomyelitis dissiminata

- Tinnitus

- Epilepsie

- Neuropathien

- Neuroborreliose

- Morbus Parkinson

- Restless legs Syndrom

- Tremor

- Extrapyramidal-motorische

- Störungen (EPS)

- Fazialis-Parese nach Therapien

- Erfahrungen mit „moderner“

Therapie bei zahlreichen

Diagnosen

nach Differenzialdiagnosen

- z.B. Kopfschmerz

- Indikation zu weiterführenden diagnostischen Maßnahmen

- stationäre Einweisungen

nach Schwierigkeiten

- „off-label“ Problematik piekonzeption sind häufig

- Aggression: Der häufigste Grund für eine stationäre Therapie besteht in der Unkenntnis möglicher Bewältigungs- und Behandlungsstrategien

- Demenz: Die meisten hausärztlichen Anfragen werden bei deutlich fortgeschrittenen Krankheitsverläufen gestellt (5)

- Zwangsstörungen: Es besteht große Unsicherheit und Verkennung insbesondere bei der Einschätzung der Ausprägung der Symptomatik.

\section{Nach Therapien}

- Psychopharmaka: Psychopharmakologische Kenntnisse gehen manchmal über die Kenntnis von Antidepressiva kaum hinaus. Eine differenzierte Anwendung von modernen Antidepressiva und Antipsychotika ist eher selten, häufig besteht immer noch ein „Fluspirilenismus“, bei psychiatrischen Alterserkrankungen ein „Melperonismus“

- Psychotherapie: Psychotherapeutische Maßnahmen werden häufig als Ausweg zu einer nicht zu vermittelnden oder erfolglosen spezifischen Psychopharmakotherapie gesehen.

\section{Nach Differenzialdiagnosen}

Bei unbefriedigenden Behandlungsergebnissen kommen Fragen nach weiterführenden Diagnoseschritten. Zum Teil werden hausärztliche diagnostische Maßnahmen rechtzeitig durch nervenärztliches Fachwissen gebahnt oder ergänzt.

\section{Nach Schwierigkeiten}

Komplikationen und Nebenwirkungen der primär eingeleiteten therapeutischen Maßnahmen führen zu einer Verunsicherung der hausärztlichen Behandlungsstrategie. Nicht selten sind Anfragen zu Therapieleitlinien bei vermeintlichen Non-Respondern.

\section{Zu den neurologischen Fragestellungen Nach Diagnosen}

- Schwindel: Häufigste Zuweisungen kommen aus dem kardiovaskulären Bereich 
- Wirbelsäulensyndrom: Chronifizierte Beschwerden mit Erschöpfung der Behandlungsoptionen stellen auch für den Nervenarzt schwierige Aufgaben dar

- Enzephalomyelitis disseminata: Schnelle Zuweisungen zwecks diagnostischer Abklärung und Erstellung von Therapiestrategien nehmen bemerkenswert zu

- Tinnitus: Häufig werden Patienten mit langjährigem Verlauf und mit frustranen Behandlungsergebnissen vorgestellt. Die Überweisung zum Nervenarzt erfolgt oft als Ultima Ratio

- Epilepsie: Häufig steht die Synkopenabklärung nach internistischer Vordiagnostik im Vordergrund

- Neuropathien: Relativ späte Anfragen nach vielseitigen Voruntersuchungen mit unbefriedigenden Anbehandlungen erschweren die erfolgreiche Anwendung nervenärztlicher Strategien

- Neuroborreliose: Fragen an den Nervenarzt zur Behandlung der Neuroborreliose werden oft erst dann gestellt, wenn die antibiotische Behandlung bereits abgeschlossen ist und wenn für den Hausarzt schwer erklärbare Symptome oder psychische Störungen auftreten. Sie umfassen sowohl diagnostische wie therapeutische Problembereiche.

- Morbus Parkinson: Die meisten Anfragen werden bei Therapiekomplikationen im späten Stadium gestellt (6)

- Restless Legs Syndrom: Schwierige Differenzialdiagnosen stehen im Vordergrund der Anfragen

- Tremor: Häufig sind Anfragen bei Non-Response unter vorbestehender anticholinerger Medikation

- Extrapyramidalmotorische Störungen (EPS): Differenzialdiagnostische Fragen und Probleme bei der Ursachenzuschreibung führen in der Regel zur Überweisung an den Nervenarzt

- Fazialisparese: Fragen nach der Notwendigkeit einer Therapie generell stehen im Vordergrund.

\section{Nach Therapien}

Erfahrungen in „moderner“ Therapie wie mit Immunglobulinen, noch nicht zugelassener Medikation, Kombinationsbehandlungen o. Ä. häufen sich. Gute Kenntnisse in „klassischen“ Therapieverfahren und die Aktualisierung moderner Behandlungsmethoden durch den Nervenarzt sind erwünscht.

\section{Nach Differenzialdiagnosen}

Die häufigste Anfrage z.B. bei Kopfschmerzen ist die zur Differentaldiagnostik in fortgeschrittenen Stadien der Erkrankung und die nach der Indikationsstellung weiterführender diagnostischer Maßnahmen. Häufig wird der Nervenarzt als Letztes gefragt, zuvor waren die Patienten bereits Orthopäden, HalsNasen-Ohrenärzten oder Augenärzten vorgestellt worden. Die Aufgabe, die dem Nervenarzt dann zugewiesen wird, ist die „Sichtung“ des oft umfangreichen Befundmaterials, um danach ein neues diagnostisches und therapeutisches Konzept zu erstellen. Vielfach wird auch darauf verwiesen, dass die Aufgabe ambulant nicht mehr zu bewältigen sei und eine stationäre Abklärung erfolgen müsse.

\section{Nach Schwierigkeiten}

Die „off-label“ Problematik bei zahlreichen Medikamenten macht zunehmende Schwierigkeiten (7). Nervenärzte werden angefragt, ob Krankenkassen die Kosten für bestimmte Therapien übernehmen, aus Unkenntnis, ob diese Therapie für den speziellen Indikationsbereich zugelassen ist. Immer wieder werden aber auch Überweisungen an den Nervenarzt ausgestellt, wenn die eingeschlagene Therapie kostenintensiv ist und das eigene Budget damit nicht belastet werden soll.

\section{Was ist aus den Anfragen der Hausärzte abzuleiten?}

Die Anfragen lassen zunächst den Schluss zu, dass sich eine Vielzahl der Patienten mit allgemeinmedizinischen Diagnosen und neuropsychiatrischen Beschwerden in hausärztlicher Betreuung befinden. Dies ist aus primärärztlicher Versorgung nicht verwunderlich und auch wünschenswert. Allerdings muss festgestellt werden, dass der Nervenarzt auch bei in sein Fachgebiet fallenden Fragestellungen oft erst spät oder gar zuletzt gefragt wird. Dabei stellt sich die Situation im großstädtischen Raum sicherlich etwas anders dar als in ländlichen Bereichen, entsprechend der Dichte der fachärztlichen Versorgung. In vertragsfachärztlichen Ballungsgebieten wie Hamburg, in denen eine nervenärztliche Diagnostik und Weiterbehandlung problemlos möglich ist, teilen sich die hausärztlichen Überweisungen der Patienten mit neuro-psychiatrischen Beschwerden je nach Schule der universitären Ausbildung, Interesse am Fachgebiet und Kontakten zu Fachärzten der Überweiser wie folgt auf, wobei eine computerausgewiesene Reihenfolge von der KV-Hamburg nicht abgefragt werden kann:

- Orthopädie

- Radiologie

- Innere Medizin

- Kardiologie

- Gastroenterologie

- Psychotherapie

- Nervenheilkunde.

Damit die eingeschlagenen $\mathrm{Be}$ handlungswege für den Überweiser überschaubar bleiben, müssen die angefragten Fachärzte inhaltlich sinnvoll kooperieren und Untersuchungsbefunde und Behandlungsergebnisse rückmelden. Der Inhalt und praktische Nutzen dieser Rückmeldungen ist aus Sicht des Nervenarztes besonders dann wichtig, wenn er, wie bisher häufig, die diagnostische Schlusslichtposition einnimmt und nicht von vornherein in das Konzept der Differenzialdiagnostik miteingebunden wird. Das heisst, dass die Reihenfolge differenzialdiagnostischer Überlegungen symptomorientiert sinnvoll, zeitgleich mit anderen Inputs und zielgerecht eingehalten werden soll. Ökonomisch bedeutet dies, eine zusammengefasste relevante Datenerhebung auf modernem Wege weiterzugeben, um schnellstmögliche fachärztlich übergreifende Therapieziele zu erreichen. Bislang bestehen nur allzu häufig kostenungünstige Zeitverluste durch unsichere 
Anbehandlungen, ärztliche Alleingänge und verwirrende Konzepte. Gerade die wichtige Früherkennung und -behandlung vieler psychiatrischer Störungen leidet hierunter. Hierdurch empfindet der Kranke eher Nebenwirkungen statt Wirkungen der Therapie, bricht sie ggf. ab, möglicherweise kommt es sogar zu unnötigen oder zu später notwendigen stationären Einweisungen, da mittlerweile die Progression der Erkrankung fortgeschritten ist. Ein solches ärztliches Vorgehen ist nicht nur für die Compliance zum Patienten nicht förderlich, sondern kann unter Umständen die Prognose der Erkrankung verschlechtern. Für die Kooperation gemeinsamer Behandlungsstrategie bei depressiv Erkrankten sei kurz ein Bespiel skizziert:

Der Einsatz von Antidepressiva bei vorliegender depressiver Störung erfolgt in der Praxis des Allgemeinmediziners nach sehr komplexen Kriterien, ähnlich wie im nervenärztlichen Setting. Allerdings ist das differenzialtherapeutische Spektrum des Hausarztes bei der Auswahl eines Antidepressivums deutlich limitierter als das des Nervenarztes, der über größere Kenntnnisse in der Indikationsstellung verfügt und auch psychopharmakologische Hintergründe berücksichtigt. Die Auswahl der Medikation richtet sich in erster Linie nach Wirksamkeit, möglichen Nebenwirkungen und Verträglichkeit. Der Einsatz von Präparaten der ersten Wahl und eventuelle Wechsel zu anderen Stoffklassen sollten begründet werden. Die Kenntnis der Wirkmechanismen der einzelnen Antidepressiva mit den Hinweisen auf pharmakologische Eigenschaften, eventuelle Nebenwirkungen, mögliche Risiken, zu erwartende Interaktionen und besondere pharmakokinetische Parameter müssen bei fachärztlichem Handeln vorausgesetzt werden. Es sollen auch weitere Argumente für den differenzialtherapeutischen Einsatz einzelner Antidepressiva bereitgehalten und Therapiestrategien in allen Behandlungsabschnitten von der Akutbehandlung bis zur Prophylaxe aufgezeigt werden. Alter und soziale Situation der Kranken müssen berücksichtigt werden. Dieser Wissensstand kann von Hausärzten nicht vorausge- setzt und verlangt werden. Aus Sicht eines niedergelasssenen Facharztes für Nervenheilkunde wird dem Allgemeinmediziner als häufigem Erstbehandler empfohlen, sinnvolle Strategien mit dem Nervenarzt gemeinsam zu erörtern und einzuleiten und das Prozedere bis zu einer eventuell notwendigen vielschichtigeren nervenfachärztlichen Therapie festzulegen.

\section{Wie kann die Verbesserung der Therapiekonzepte aussehen?}

Eine ökonomische, moderne erste Einschätzung von geschilderten neuropsychiatrisch relevanten Krankheitssymptomen im ambulanten hausärztlichen Bereich könnte darin bestehen, dass einer vorgegebenen Labordiagnostik und allgemeinmedizinischen Basisuntersuchung eine nervenärztliche Diagnostik direkt folgt. Die Ergebnisse dieser ersten hausärztlichen Behandlungsschritte stellen den „Filter“ für ggf. weiterführende, differenzialdiagnostische Maßnahmen dar bzw. sind der Beginn einer gemeinsamen Behandlungsstrategie. Hierbei muss auch der Nervenarzt zu einer größeren Flexibilität seines Praxismanagement aufgefordert werden.

\section{Übergreifende Fortbildung}

Wie immer auch die politische Entwicklung in Zukunft die Strukturen ambulanter Versorgung neuropsychiatrischer Patienten beeinflusst, wird ein gemeinsames Know how, z.B. hinsichtlich der Therapiestrategien bei psychiatrischen und neurologischen Krankheitsbildern, Ergebnis vernetzter, interdisziplinärer Fortbildungen sein, in das Wissen aus Universität, fachärztlicher und allgemeinmedizinischer Kompetenz zusammenfließen müssen.

\section{Gemeinsames Arbeiten}

Als ich vor 18 Jahren an einem ersten Seminar zur Praxisführung teilnahm, hörte ich den Referenten sagen, die ideale Praxisgemeinschaft sei die zwischen einem Allgemeinmediziner und einem Nervenarzt. Die langjährige Betreuung, die umfassende Kenntnis der Leiden und die Vielschichtigkeit der Krankheitssymptome der Patienten könnten tatsächlich unsere beiden Arztgruppen im medizinischen Wissen und Handeln näher aneinander rücken lassen.

Currently, we have no reliable data sources providing solid statistical information on the referral practice between general practitioners and specialists. In the present article, the subjective experience of a neurologist with queries by family doctors on neuropsychiatric illnesses is described. It is shown that the neurologist is often consulted for advice on diagnosis and treatment only at a late stage, although both could profit from an improvement in cooperation. Further education on a networked and interdisciplinary basis is urgently required to enable the knowledge generated in universities and within the competencies of specialists' and general practitioners to be pooled and shared.

\section{Literatur}

1. Wittchen HU, Müller N, Storz S. Psychische Störungen: Häufigkeit, psychosoziale Beeinträchtigungen und Zusammenhänge mit körperlichen Erkrankungen. Gesundheitswesen 1998; 60: 95-100

2. Volz HP, Naber D, Deckert C. Die Langzeittherapie der Schizophrenie. psychoneuro 2003; 29: 118-122

3. Rüther E. Schizophrenietherapie im Wandel der Zeit. psycho 2002; 28: 539-543 4. Wittchen HU, Krause P, Hoyer J, Beesdo K, Jacobi F, Höfler M, Winter S. Prävalenz und Korrelate Generalisierter Angststörungen in der Allgemeinarztpraxis. Fortschritte der Medizin 2001; 119: 17-25

5. Ihl R. Zwischen gesundem Altern und Demenz. DNP 2003: 3; 43-46

6 . Lemke MR. Depression bei M. Parkinson. DNP 2003; 7/8: 36-38

7. Dobmeier M. Antikonvulsiva. Stellenwert in der Behandlung und Prävention von bipolaren Störungen. Krankenhauspsychiatrie 2002; 13: 44-48

Korrespondenzadresse: Dr. med. Christian Deckert Arzt für Neurologie und Psychiatrie Moorweidenstr. 12 20148 Hamburg e-mail christian@dr-deckert.de www.dr-deckert.de 